\title{
Analysis of the Power Drives of Terrain Forklifts
}

\author{
Georgi Valkov \\ Department of Design and Technology \\ Balkancar Record Co. \\ 17 Kuklensko Shose str., 4004, Plovdiv, \\ Bulgaria \\ techno@balkancar-record.com
}

\begin{abstract}
The main subject of this publication is to provides a description of terrain forklifts, their design and specific features. The main mechanical, hydrodynamic and hydrostatic types of power drives and their application in terrain forklifts are presented herein. This article includes an analysis of the power transmissions drive systems of terrain forklifts. The most important trends in the development of the terrain forklifts have also been presented.
\end{abstract}

Keywords-Terrain forklifts, mechanical, hydrodynamic and hydrostatic power drives.

\section{INTRODUCTION}

The boom in the development and deployment of terrain forklifts which has lasted for several decades continues to be one of the current trends in the design of forklifts worldwide. These forklift trucks are designed to carry out lifting and transport operations in agriculture and forestry, in construction, at open storage sites, waste recycling sites, ports, military fields, etc. Terrain forklifts are designed to operate on both hard and soft roads characterized by significant unevenness and different physical and technical properties of the soil (structure, humidity and density). These conditions determine the nature of the interaction between the forklift drive system and the road and significantly influence its traction properties, which has a direct impact on the productivity and fuel economy of the forklift.

The towing-traction properties of the forklift truck characterize its traction capability and its passability when moving on different types of roads.

Substantial influence on the towing-traction properties and sustainability of forklift trucks is the weight of the machine, the load and its distribution on the axles, the engine power, the forklift truck composition design, the kinematic power transmission design, the wheel formula, the type and the technical characteristics of the wheel drive [1], [2], [4] - [7].

The great variety of composition designs and constructive developments of these machines and their use in different soil conditions require the search for optimum conditions in the compilation of mathematical models and the development of an optimization program.

Terrain forklifts or "high passability forklifts" and "off-

\section{Valyo Nikolov}

Department of Transport and Aviation

Equipment and Technologies, Technical

University of Sofia, Plovdiv Branch

25 Tsanko Dyustabanov str., 4000

Plovdiv, Bulgaria

vnikolov@tu-plovdiv.bg

road forklifts".as are frequently mentioned in literature.

The production of terrain forklifts and special work equipment for them has shown a continuous tendency for increasing the volumes in recent years. The reason for that is the constant increas in the need for mechanization of loading and unloading and some other types of work, as well as the need of increased productivity in construction, forestry and agriculture and other specialized terrains.

Geographically, the production of these vehicles, except in Europe and North America, where the major suppliers of this equipment are the well-known "Manitou", AUSA, "Case", JSB, "Agrimac" and other ones, has been transferred to Asia in recent years. Companies such as "Maximal", "Heli", "Hangcha" and others have very successfully entered this market.

On the territory of the Republic of Bulgaria to this date the companies that produce this type of equipment are "Balkancar Record" Co., IPO and "Herku - Milko Tanev".

The main type of mass-produced forklift trucks feature a working body of lifting mast unit [1], [5].

Since the technical and advertising literature often refers to the concepts of "high passability forklifts“ and „off-road forklifts", it should be borne in mind that there are significant differences between these two types of forklifts.

\section{High Passability Forklift Trucks}

The most common feature of these trucks is the classic computational design - engine, gearbox and front drive axle. The front wheels are large in size and the rear wheels are with considerably smaller size - see Fig. 1 [9].

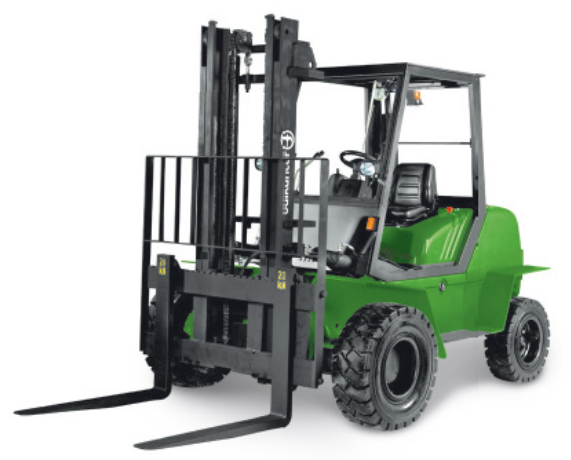

Fig. 1. Forklift with the "classic" design - engine, gearbox and front drive wheels.

Print ISSN 1691-5402

Online ISSN 2256-070X

http://dx.doi.org/10.17770/etr2019vol3.4197

(C) 2019 Georgi Valkov, Valyo Nikolov.

Published by Rezekne Academy of Technologies.

This is an open access article under the Creative Commons Attribution 4.0 International License. 
Forklifts produced according to this design primarily feature wheel formula $4 \times 2$. The capacity of these forklifts ranges from 1,000 to $10,000 \mathrm{~kg}$. Machines featuring wheel formula $4 \times 4$ are also produced. Using this wheel formula the transmission ratio of the front and rear drive axles varies due to the different diameters of the front and rear wheels. In addition to this computational design, a splitting distribution gearbox can also be included, and the rear steering axle can also be a drive axle.

All standardized attachments, which can be mounted on the forklifts with universal forklifts mast may also be used for terrain forklifts with the main working body of lifting mast unit. In addition, the following specialized working units are also produced: various types of baskets - sand, ballast, concrete, for various agricultural production and others. An excavator can also be used, which is reversed in the direction of movement above the engine bonnet at the back of the machine with a separate workspace for the operator [5].

Due to the high unification of the forklift trucks of the $4 \times 2$ family with industrial forklift trucks, their power transmission design is the same: a rear-end engine, a hydrodynamic gearbox, a twin cardan coupling joint, a front drive axle and a rear steering axle. The difference only involves the use of a larger diameter wheels and lowpressure wide-profile tires.

For forklifts with wheel formula $4 \times 4$, the distribution gearbox is rigidly mounted to the transmission or to any of the drive axles. There are two cardan coupling between the distribution gearboxes and the front and back drive axles. The rear wheels are smaller in size than the front wheels to achieve better maneuverability. All of the above-mentioned specialized mast attachments used on forklift with $4 \times 2$ wheel formula can also be used for this type. The advantage of these machines is lower vibration activity at driver's seat and compact operating parameters and dimensions. Mostly hydrodynamic and hydrostatic transmissions are used with this wheel formula.

The most important features of the above- mentioned forklift trucks are:

- In most computational design, the drive and steering axles are of the portal type - to enhance road clearance and their being stronger;

- In many forklift truck models, inter-wheel differentials are not blockable.

- Wheel forklifts featuring wheel formula $4 \times 2$ are without splitting distribution gearboxes.

- Regarding forklift trucks with $4 \times 4$ wheel formula, most often have a $100 \%$ front drive axle locking ability and a $45 \%$ automatic locking on the rear drive axle.

- In the most gearbox designs for forklift trucks with wheel formula $4 \times 4$ wheelbase differentials are not placed;

- In the latest models one of the cardan couplings is removed by attaching a distribution box to the front or rear axles;

- In recent years, the hydrostatic transmission has been increasingly used with a hydro-motor coupled to the gearbox.

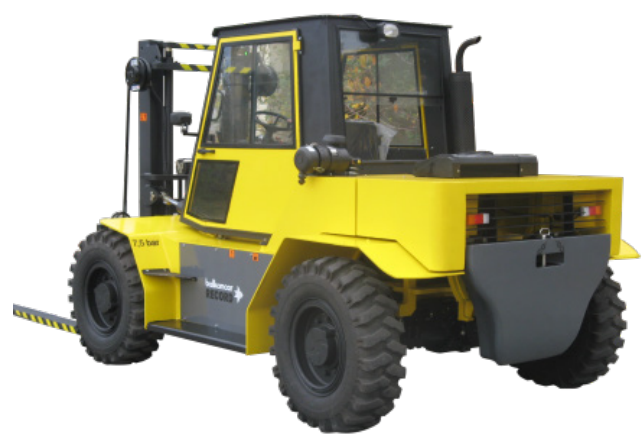

Fig. 2. Off-road forklift.

Insufficient passability of the above-described types of vehicles due to the relatively small and narrow rear wheels and insufficient universality when working with a lifting equipment, regardless of the specialized changeable mast attachments, entailed the development and production of three new types of terrain forklifts with wheel formula $4 \times 4$ at the end of the last century. They feature identically sized front and rear wheels that are fitted with wide-profile tires of low air pressure therein. Such machines are shown in Fig. 2 [9].

\section{OFF-ROAD FORKLIFTS}

The first type forklift trucks is with two frame joints connected (articulated forklift trucks), a lifting mast and rapidly changing different types of lifting mast attachments.

The second type is complete frame forklifts, two drive axles (of which two or one is used for steering) and a lifting mast.

The third type are complete frame forklifts, two drive axles (of which two or one is for steering) and one specialized working equipment unit - a telescopically extending boom, but with greater capabilities for using different attachments.

The turning of articulated forklift trucks (those of the first type) is done by turning the wheels on the front of the frame to the rear. Axles are only drive axles, which are at lower cost than the drive axles with steering wheels. The frame is more complicated and consists of three parts front, rear and articulation. Adjustment of the machine on uneven terrain occurs in two ways.

The first one, which is also most commonly used, is an articulation with only one degree of freedom (rotation around the vertical axis and a swinging rear axle /rotation around the horizontal axis).

The second way is through an articulation with two degrees of freedom - one turning around the vertical axis and one turning around the horizontal axis. In this case, the entire rear end of the frame rotates in relation to the front. Forklift trucks pivoted articulated $4 \times 4$ were launched for the first time by the company "Omfort", UK. They received a wide distribution for a short time. The most typical thing concerning this type of machine is the use of a large number of different work attachments, that can be changed only by the driver for a short time using built-in quick-ly changeable devices. The following working equipments are placed on the chassis: a mast, a loader with forks, an excavator, a dump, a bulldozer, a hydraulic hammer - breaker, a screwdriver (for drilling 
holes in the soil), etc. In addition to them, all of the abovementioned removable work attachment equipment can be fitted.

The power drive transmission of articulated machines is as described above: an engine featuring mounted transmission box, a distribution gearbox, and a cardan joint between it and the drive axles.

Turning of forklift trucks with a full frame, with lifting equipment or telescopic-extending boom (those of the second and third type) is done by turning the wheels of the rear drive axle. Due to the constraints imposed by the cardan couplings and the large wheel sizes, the turn radius of this type of machine is relatively large. Due to the need for increasing their maneuverability, forklifts with both axles constitutes driving and steering units have been developed. The adjustment of the machines on the uneven ground is done by a swinging rear axle (rotation around the longitudinal axis). The main advantage of this type of forklift trucks is expressed in two aspects: when using a boom, its pulling forward allows for the most appropriate loading and unloading of means of transport with large loading platforms. The boom makes it possible to achieve high lifting heights of the load - up to $12-13 \mathrm{~m}$. This allows building materials to be delivered up to 3-4 storey buildings in farm yards or out-of-town settlements. The boom can be fitted with all attachments such as those for articulated forklifts, except for the excavator.

The power drives of forklift trucks with a full frame, a lifting unit or a telescopic-exiting boom in most models feature distribution boxes coupled to one of the axle.

Articulated and complete frame forklift trucks are mainly produced with hydropower transmissions, with a hydro motor attached to the transmission box or with a hydro-dynamic transmission and distribution gearbox. The axles are mainly portal type with locking inter-wheel differentials and multi-disc brakes operating in oil on both sides of the first stage of the main transmission. Wheelbase differentials are rarely incorporated in some models.

Off-road forklifts are available at the following lifting capacity: 1,$500 ; 2,500 ; 3,500 ; 4,000 ; 5,000 ; 6,000 ; 7,000$; 8,000 , and $10,000 \mathrm{~kg}$.

In summary, the main concepts on which the off-road forklift trucks are developed are the following:

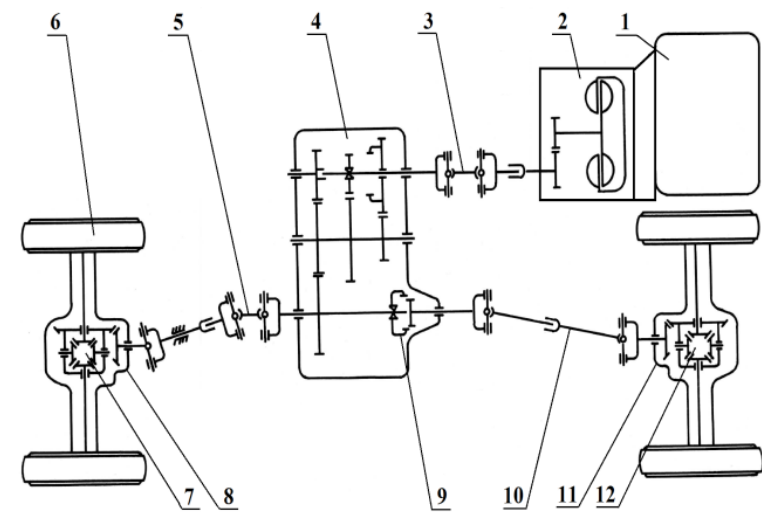

Fig. 3. Power drive design of the articulated forklift family with two drive axles, where: 1 . Internal combustion engine (ICE) 2. Hydrodynamic gearbox; 3. Cardan coupling; 4. Two-stage distribution gearbox; 5. Cardan coupling with intermediate bearing; 6 . Wheel drive; 7. Differential of front drive axle; 8. Front drive axle; 9. Coupling for enabling/disabling of the rear drive axle; 10. Cardan coupling; 11. Rear drive axle; 12. Differential of the rear drive axle.
The power (engine), transmission, and drive axle design of articulated frame and complete frame trucks have virtually no difference. The power drive design of the articulated forklift range is shown in Fig.3 [9].

The power drive design of the complete frame forklift family with two drive and steering axles is shown on Fig. 4 [9]. It is essentially the same as the one regarding articulated forklifts. The main differences are - the cardan coupling /8/ to the front axle, which is two-carded without intermediate bearing, the presence of synchronous cardan couplings /9/ in both drive and steering axles, as well as their construction.

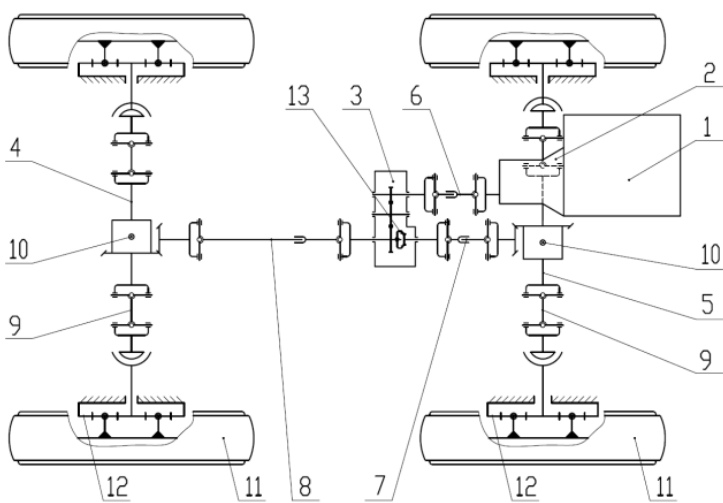

Fig. 4.Power drive design of the complete frame forklift family with two drive and steering axles, where: 1 . ICE; 2. Hydrodynamic gearbox; 3. One-stage distribution gearbox; 4. Front drive axle; 5. Rear drive axle; 6. Cardan coupling; 7. Cardan coupling; 8. Cardan coupling ; 9. Synchronous cardan coupling; 10. Differential of front and rear drive axles; 11. Wheel drive; 12. Endwheel reduction gearbox; 13. Coupling for enabling/disabling of the rear drive axle.

Transmission hydrodynamic gearboxes are usually produced with two forward gears and two rear gears or three forward and three reverse gears.

The distribution gearboxes are single-stage or twostage in most cases without a wheelbase differential. They only have a coupling to shut off torque transmission transfer to the rear axle at the driver's request.

The use of cardan couplings with telescopic extension similar to the one used in many lorries.

The wheels of all the above-mentioned forklifts feature wide-profile, large diameter and lower pressure. The frames of the three types of forklift trucks differ considerably in their construction.

The hinged articulated forklift's frames consist of three parts - front, rear and articulation. The front part is a frame construction of steel profiles. Attached to it is the work body, the front axle, the cab with the controls and the protective roof. The axle is rigid handing to the front of the frame with bolts. The rear part is a box-like structure made of thick sheets of steel and profiles. The engine with transmission gearbox, distribution gearbox, the rear axle and the fuel and oil tanks are attached to it. The rear axle is rigid handing also to the frame. The fitting of a forklift on the ground is done by swinging the rear frame to the front by using an articulation with one or two degrees of freedom.

The frames of the forklift trucks with two drive and steering axles are considerably simpler. They are two longitudinal sheets of thick steel, connected with 3 or 4 cross beams. The front axle is rigid handing to the frame 
and the rear of the swing by means of 2 metalrubber bearings and two rubber springs or an axle.

The servosteering of off-road forklifts is hydrostatic.

Their service brakes are drum brakes for some of the machines. Nowadays, oil-friction discs are predominant, acting like the others on all wheels. The brake systems feature a brake booster for trucks with a load capacity of over $4,000 \mathrm{~kg}$. The hydraulic braking of lower capacity loaders does not differ from that of universal forklifts and is carried out with unified components.

The parking brakes of all machines are located on the front drive axle. The braking action is mechanical. In the newer axles the handbrake is negative, it is released by an electro-hydraulic unit.

A hydraulic unit is used to trigger all the specific working equipment and operations. Also, it is used for the steering of the forklift truck. Hydraulic distributors for this purpose are multi-sectional, with manual or joystick control.

Off-road forklifts are designed to work with several types of quick-ly changeable work attachments. For the quick change, by the driver only, of the different operating organs, special attachment devices have been developed.

For articulated forklifts, quick-ly change able attachments are used: a dumper, pliers, a bulldozer, a hydraulic hammer, a screwdriver, a bucket, a grapple manure loader, a grapple for lengthy materials.

For the whole frame forklift family with two drive and steering axles, a telescopically extending boom has been developed for reaching high lifting heights.

\section{POwER Drives of Terrain Forklifts}

Since the power transmission design for type $4 \times 2$ forklifts is the same as the one of the universal forklift trucks, we will look at the power drive only of the $4 \times 4$ wheel formula forklifts.

In the forklift trucks featuring wheel formula $4 \times 4$, a variety of construction designs is used for their power transmissions. Regardless of their large variety depending on how the torque is transmitted to the front and rear drive wheels, power transmissions can be subdivided into mechanical, hydrodynamic and hydrostatic. In the analysis of existing constructions, the main attention is focused on the kinematic and power links between the individual drive motors and their mutual influence on the design and performance of the machines.

\section{A. Mechanical power transmissions}

Mechanical power transmissions are widely used in cars, tractors, trucks, dumpers and other transport machines. Compared with other types of power transmissions, they are characterized by simplicity, reliability in operation and high efficiency, because they do not convert energy from one type to another. In contemporary technological development, however, due to such drawbacks as stepping, manual gear shifting, large number of levers and pedals, large size and weight, etc., they increasingly fall back on other types of power transmissions or are used in combination with them.

Concerning vehicles featuring the $4 \times 4$ wheel formula, there is a wide variety of composition designs which are determined by the design of the machines, the adopted construction of other elements and systems such as suspension, steering system, etc. The most important aspect of the passability and traction properties of the machines is the distribution of the engine's power between the individual wheels and the axles.

Mechanical power transmission is mainly used in high passability forklift trucks. The most commonly used composition design is the so-called "I-shaped".

I-shaped power transmissions (Fig. 5) [2] have been more widely manufactured on $4 \times 4$ machines due to the smaller number of cardan couplings and gearboxes and their cheaper production. In this case, the torque from the engine / / via the transmission box / $/$ and the distribution gearbox $/ 3 /$ is transmitted to the main differentials of the front and rear axle $/ 4 /$ and by inter-wheel differentials to the drive wheels $/ 5 /$

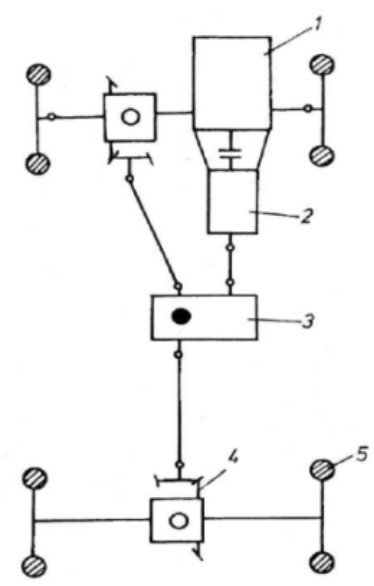

Fig. 5. Design of I-shaped power transmissions.

The design of Fig. 5 is most common in high passability forklift trucks with a small lifting capacity of 1,000 to $1,500 \mathrm{~kg}$. The front and rear axles are permanently connected via a symmetrical differential lock with a manual locking capability. Between the wheels of the two axles there is almost always a differential connection.

In order to avoid the drawbacks of mechanical power transmission and its limited use in terrain forklifts featuring low lifting capacity, it is necessary to use other power transmissions in the machines with higher lifting capacity - hydrodynamic power transmission.

\section{B. Hydrodynamic power transmissions.}

Hydrodynamic power transmissions are used in both rough terrain and off-road vehicles. They are used in forklifts with a lifting capacity of over $2,500 \mathrm{~kg}$. For all machines in the hydrodynamic transmission boxes, after the hydrotransformer, a mechanical gearbox featuring 1, 2 or 3 forward and reverse gears is used.

Fig. 3 shows a hydrodynamic power transmission design of off-road forklifts with a single-speed hydrodynamic gearbox, two-stage distribution box and articulated chassis produced in the 1980s in "Balkancar Record" Co. [9].

Fig. 6 [9] show the diagram of the highway DV80/40 $4 \times 4$ HD forklift with lifting capacity of $8,000 \mathrm{~kg}$ produced by "Balkancar Record" Co. The transmission box /2/ is 
coupled to the distribution gearbox /3/. The distribution box is of the T12000 type and has three stages. It can enable/disable the torque transmission to the rear axle via the coupling /8/. The main transmission consists of the cone pair $/ 4 /$, the end wheel gearboxes $/ 5 /$ are exported to the wheel drive $/ 6 /$. There is one output for PTO $/ 7 /$ which is used for a power output for propulsion of hydraulic pumps on the gearbox $/ 2 /$. There is no differential connection between the front and rear axles. The front differential /9/ has the option of $100 \%$ forced locking, and the rear axle $/ 10 /$ is $45 \%$ self-locking. A feature of the design is the adopted construction with two driving and one rear steering axle. The front axle is rigid handing to the frame and the rear axle is swinging on a specially designed shaft.

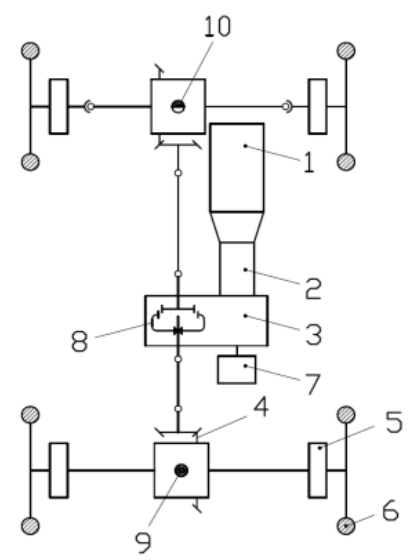

Fig. 6. Design of power transmissions of DV80/40 4x4 HD forklift with lifting capacity of $8,000 \mathrm{~kg}$.

From the schematic view of the mechanical and hydrodynamic power drive transmissions of $4 \times 4$ forklifts it is evident that the most applications are I-shaped using different types of wheelbase and inter-wheel differentials. With the use of regular inter-wheel differentials and the same road traction conditions of the left and right wheels, the best performance and patency characteristics of the machine are provided both in lineal motion and when turning. When using forced manual or hydraulic locking, the most complete adhesion of the wheels to the road can be achieved. Under various traction conditions of the left and right wheels, it is sometimes difficult for the driver to determine the optimal solution and often requires the locking to be switched on and off. Inter-wheel differentials are also used in self-locking, which have a common lock factor ranging from 1.6 to 6 , which provides some automaticity, but worsens the steering control during turns.

Wheelbase differentials are rarely used and they are common with option for manual locking. Most popular are gearboxes with coupling for manual or hydraulic enabling and disabling of the lock of the rear axle when wheelbase differential is not used.

\section{Hydrostatic power transmissions.}

A great interest in the hydrostatic power transmissions of terrain forklifts is shown by all the design teams dealing with this technique. This is determined by the rapid development of scientific and technological progress, the implementation of modern technologies, the incorporation of a number of types of hydromachines in serial production, on the one hand, and the need for highperformance transport machines allowing for complex mechanization and automation of the basic loading and unloading work they perform, on the other hand.

The most important advantages of hydrostatic power transmissions for terrain forklifts that can be highlighted involve:

1. Possibility for stepless adjustment of the speed of movement in quite a wide range.

2. High compactness, small mass and overall dimensions, which reduce the machines weight.

3. Low inertia, ensuring good dynamic properties of the machines, including fast reverse movement.

4. Easy automation of power transmission.

5. Independent location of the power transmission individual elements, which enables to be placed in the most convenient positions for the composition design. Great structural flexibility for design engineers.

6. Reliable protection of the motor and transmission from overloading by incorporating safety valves.

7. Use of unified assembly units (pumps, hydromotors, valves, distributors, filters, etc.) to reduce production cost and to facilitate the operation and repair of machines.

It should be taken into account that in practice the effective implementation of the advantages of the hydrostatic power drive transmission is hampered by the following disadvantages:

1. Significant dependence on the performance and reliability of the power transmission from the ambient temperature and the viscosity of the working fluid, respectively.

2. Existence of internal and external leakage due to airtightness of the system, requiring compensation and causing volume losses.

3. Increased requirements for the operational properties of working fluids - the need for constant filtration to remove the dirt. The periods between machine servicing are reduced.

4. Increased requirements for the used materials, quality of workmanship and assembly of the part of the hydraulic equipment.

5. Need for highly qualified personnel and personal responsibility in operating and servicing machinery.

There is a wide variety in the hydrostatic design of hydropower transmissions for terrain forklifts. It should be noted that they are used in both rough terrain and offroad vehicles, whether the wheel formula is $4 \times 2$ or $4 \times 4$. For example, Fig. 7 [10] shows the hydropower driving of a high passability forklift model MSI 40 of "Manitou" featuring a wheel formula of $4 \times 2$ and a lifting capacity of $4,000 \mathrm{~kg}$. 


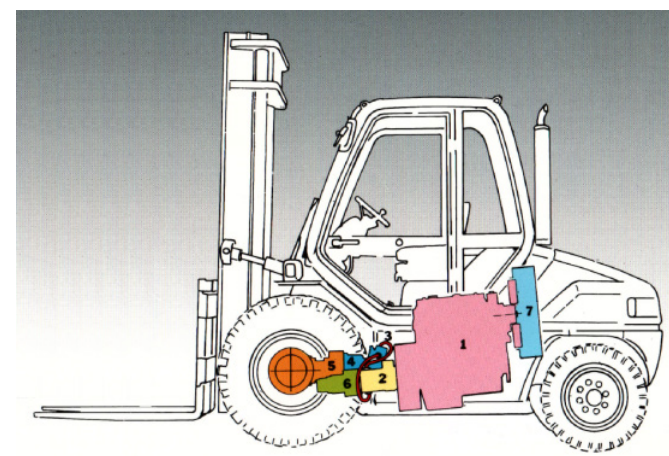

Fig. 7. Hydropower drive of a forklift, where: 1. ICE; 2. Hydrostatic axial pump; 3. Flexible high pressure hoses; 4. Hydraulic motor; 5. Drive axle; 6. Operating hydraulic toothed pump; 7. Cooler.

Adjustment of the speed of movement can be done smoothly by varying the pump productivity or the working volume of the hydromotor, or both together, and also stepwise by using composite hydromotors, switching the same from sequential coupling to parallel, etc.

Using an adjustable hydromotor and a non-adjustable pump, a transmission with a characteristic close to the ideal is obtained, but an increase in the volume and size of the hydromotor is necessary, making this circuit inadequate.

Regarding terrain forklifts, the most common hydrostatic design is the following: one adjustable pump and one adjustable high-speed motor shown in Fig. 8. [9] This design has been successfully realized in the DV80/ $4 \times 4$ off-road forklift truck of "Balkancar Record" Co., shown in Fig. 2.

With such a movement speed control, the range of high-efficiency operation of the machine is expanded, but since the mechanical part of the transmission is retained, the internal losses from friction, and other, inside the transmission are also included. This design achieves complete unification with mechanical power transmissions since they achieve it by replacing the transmission box with a hydraulic power transmission.

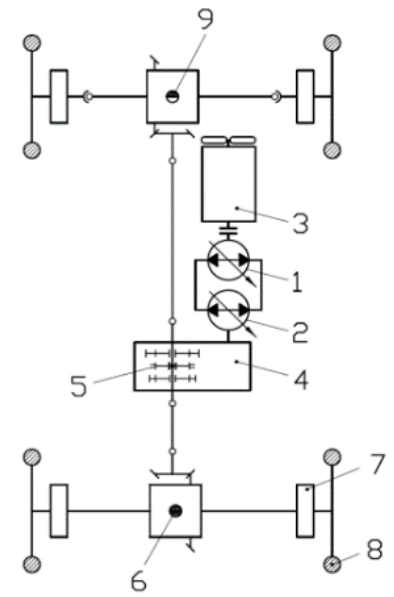

Fig. 8. Design of hydrostatic power transmissions of a forklift, where: 1. Adjustable hydrostatic pump; 2. Adjustable hydromotor; 3.

ICE; 4. Two-stage distribution gearbox with hydraulic gearshift; 5. Coupling clutches for shifting quick and slow speed; 6 .

Front differential with possibility for $100 \%$ forced locking; 7 . Endwheel reduction gearbox; 8. Wheel drive; 9. Rear differential $45 \%$ self-locking.
Another variation of the hydrostatic driving for a forklift $4 \times 4$ is shown in Figure 9 [9]. It is distinguished by its simplicity, a small number of composite units (hence low cost) and high efficiency. The ICE / $1 /$ is traditionally located at the rear part of the machine, but its fan does not blow back to the radiator like all forklift trucks - it sucks air through the radiator in front of it similar to cars and tractors. The adjustable pump $/ 2$ / is connected via an elastic coupling to the engine flywheel and the direction of movement of the machine is to be considered. The adjustable hydromotor $/ 3 /$ is connected to the rear side of the main transmission $/ 5 /$ of the rear axle via a gearbox /4/. The main gear distributor is a worm - worm gear and is connected to the main transmission of the front axle by means of a cardan coupling shaft /7/. Both axles are drive and steering axles with inter wheels lockable differentials /6/.

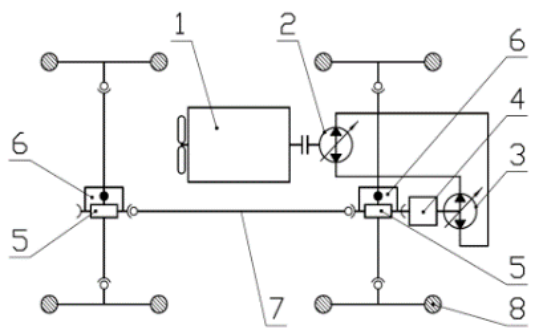

Fig. 9. Design of hydrostatic power transmissions of a forklift.

In this design there is no wheelbase differential, the axels do not have end wheel reduction gearbox and it is used on terrain forklift trucks with telescopic boom and small load capacities of 1,000 to $1,500 \mathrm{~kg}$.

Fig. 10 shows a diagram with 4 traction motors and one adjustable pump, used accidentally in terrain forklifts, mainly in the forestry industry [2].

Hydromotors $/ 3 /$ are mounted in the wheel drive /1/ together with the wheel gearboxes $/ 2 /$. The moving speed control is effected smoothly by varying the pump output /4/. The hydromotors are connected in parallel by a pilotcontrolled flow divider $/ 5 /$ between the front and rear axles. This type of connection, ensures synchronization of the peripheral speeds of the front and rear axles and locking of the differential link between the axles. The flow distribution between the two axles depends on the divider characteristic. An ideal divider is the one in which the ratio of front and rear axles flows is a constant value under all operating conditions. This composition design, as well as the independent suspension of the wheel drive, provides a high passability for all road conditions. A major drawback is its high cost. 


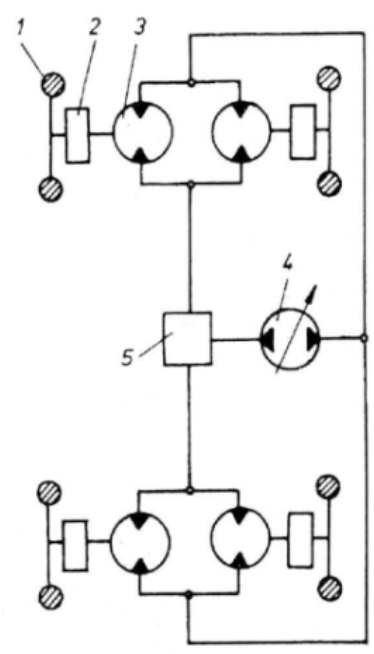

Fig. 10. Design of hydrostatic power transmissions of a forklift.

From the analyzed hydro power transmissions for $4 \mathrm{x} 4$ wheel formula forklift trucks, the following characteristic trends can develop:

1. Use high-performance and high-speed hydromotors that are integrated into the distribution gearboxes or embedded in the wheels of the machines and contribute to the simplification of the computational design.

2. Wide application finds mass-produced high-speed hydromotors that are built into the wheels via gearboxes. They are preferred because of the higher reliability and techno-economic performance compared to the high-volume ones, which are still considered special purpose products.

3. Maximum unification of mechanical transmissions - Hydrostatic transmissions are performed with a monolock hydraulic power transmission mounted instead of a gearbox.

\section{Analysis of the Power Drive Transmissions of} TERRAIN FORKLIFTS

The analysis of power transmissions in modern $4 \times 4$ terrain forklifts shows that widespread distribution finds the differential driving between the wheels and its locking to a certain extent. Differential driving between the axles is more rarely used.

Differential driving is characterized by the use of differentials in all units of division of power flow from the transmission in the forklift truck with wheels [3].

Differential drive can be symmetrical and asymmetrical. The condition to be satisfied by the symmetrical drive is in the absence of internal friction, the torque distribution should be equal between the drive shafts, and regarding the case of cartridge locking and forced rotation of one of these shafts, the other shaft should be rotating in the reverse direction at the same angular velocity. The symmetrical drive ensures the equality of provided torque on all wheels regardless of the moving conditions.

The asymmetric drive provides unequal torque distribution along the shafts. In practice, the nonsymmetric differential distributes the torques in proportion to the traction weight of each drive axle, and when the cartridge is locked, the drive shafts rotate at a different angular velocity.

The locked drive is characterized by a rigid mechanical connection between all of the drive wheels. The condition to be fulfilled with it is equality of the angular speeds of all wheels.

The differential driving to the two wheels does not allow for the full utilization of the potential driving torque force of drive axle to be fully utilized, thereby reducing the towing-cohesion properties of the machine. This is observed when the load on the wheels is redistributed when the machine is operating on a transverse slope.

In $4 \times 4$ terrain forklifts inter-wheel and lesser wheelbase differentials are used with forced or automatic locking. Wheelbase differentials are mounted in distribution boxes which mainly feature differential or locked action.

Inter-wheel differentials enable the drive wheels on one axle to rotate at different angular speeds, which provide motion during turning, on uneven road or when wheels feature different radiuses. This removes the slipping and skidding of the wheels. But, as it is known, the differential divides the torque evenly between the two semi-axis. This property of the differential when driving on a transverse slope, as well as under other conditions, reduces the propelling force of the machine. Various devices for locking the differential are used to overcome this disadvantage.

Wheelbase differentials are used in the $4 \mathrm{x} 4$ forklift for inter-wheel drive of the drive wheels. In the differential drive between the angular speeds of the axle of the wheeled forklift, there is no definite variable ratio similar the locked drive. However, in the case of a locked drive, it is possible to generate parasitic circulating power due to the presence of a closed power loop and a kinematic inconsistency in the different modes of movement of the machine.

Parasitic circulating power in closed power flow occurs under construction and operational factors. The construction factors include the type of drive system and type of wheel drive, different rolling radiuses, the type of power transmission, the vertical load on the drive wheel and its distribution, the size of the longitudinal and transverse base, etc. The operational factors include varying wear of tire treads, varying tire pressure, the different road traction due to the diverse physical and mechanical properties of the soil, etc.

The resulting parasitic circulating power results in deterioration of the traction-coupled properties of the forklift, the possibility of breakage in the power drive transmission and the wear of the drive wheels.

The wheelbase axle distributes the torque between the front and rear drive wheels in proportion to the traction weight of the respective axle and enables the wheels to rotate at different angular speeds. As a result of which there is no kinematic discontinuity and the occurrence of parasitic circulating power. But the wheelbase differential is inherent in the same shortcomings that were pointed out in the inter-wheel differential. For this reason, it also provides for the incorporation of devices for forced or automatic locking of the differential. The construction of a self-locking wheelbase, installed in the distribution gearbox of a $\mathrm{CH} 200-\mathrm{CH} 250$ series forklift of AUSA, is 
marketed as "COMPEN System" [8].

In practice, it is a self-locking conical differential with friction discs, the crown is driven by a chain, which in turn is driven by a chain gear connected to the hydromotor.

In recent developments of terrain forklifts, an everwider application finds the use of "Smart Drive CT" modules similar to those shown in [11].

This module provides an electronic solution for controlling the entire power transmission drive of terrain forklifts, most commonly hydrostatic or hydro-dynamic. It selects the most optimal drive motion mode and manages the traction efforts at any moment of the vehicle [11].

\section{CONClusions}

From the study of the kinematic designs and constructions of forklift power transmissions, the following conclusions and suggestions could be pointed out:

1. Power transmissions of forklifts are mechanical, hydrodynamic and hydrostatic with a wide variety of kinematic and power drive transmission to the individual drive wheels.

2. Depending on the drive design (locked, unlocked and differential) of the drive wheels, $4 \times 4$ forklift trucks could feature the following motion modes: drive, driven, brake, neutral or free travel drive.

3. The most of existing power drive transmissions of $4 \times 4$ terrain forklifts are implemented using distributor gearboxes with locking action, which enables the occurrence of circulating parasitic power in the power transmission or incomplete use of potential tractioncohesion properties on the machine.

4. In the power transmission design of forklifts featuring wheel formula $4 \times 4$, different mechanisms and devices are incorporated between the two drive axles (inter axle differentials with or without locking devices, distribution gearboxes, cardan coupling shafts, etc.), part of which are permanently working without locking and others include manual or automatic locking depending on the load and movement modes.

5. The great variety of $4 \times 4$ terrain forklift composition designs and the power transmissions thereof, as well as their operation in various road and production conditions, require that optimal solutions be sought by developing mathematical models and programs to optimize their parameters.

\section{ACKNOWLEDGMENTS}

This work was supported by the European Regional Development Fund within the OP "Science and Education for Smart Growth 2014-2020", ProjectCompetence Centre "Smart Mechatronic, Eco- And Energy Saving Systems And Technologies“, № BG05M2OP001-1.002-0023.

\section{REFERENCES}

[1] I. Evtimov, Electric and motor forklift trucks. University of Ruse, Ruse, Bulgaria, 2014

[2] D. Katsov and Y. Dimitrov, Wheeled and tracked machines. Technical University of Sofia, Plovdiv Branch, Plovdiv, Bulgaria, 2005.

[3] K. Stepanova and A. Lafarov, Blocking differentials of trucks. Mashgiz, Moscow, Russia, 1960

[4] D. Katsov, D. Hlebarski and S. Taneva, Transmissions of automobiles. Arena Print, Plovdiv, Bulgaria, 2018.

[5] G. Valkov and V. Nikolov, „Classification of terrain forklifts”. $7^{\text {th }}$ international scientific conference TECHSYS 2018, 17-19 May 2018, Plovdiv, Bulgaria, Proceedings, pp. 253-257, [Online]. Available: http://techsys.tu-plovdiv.bg/files/TECHSYS 2018 Proceedings.pdf, [Accessed: July 15, 2018].

[6] I. Iliev and V. Nikolov, "System for automatic stability of forklift trucks". Patent BG 62225 B1, November 15, 2007.

[7] I. Iliev and V. Nikolov, "System for control of the operations of forklift trucks". Patent BG 62226 B1, November 15, 2007.

[8] https://www.ausa.com/en-gb/forklifts/ [Accessed March 19, 2019]

[9] https://balkancar-record.com/en-US/Products/Forklifts, [Accessed March 19, 2019].

[10] https://www.manitou.com/en/ [Accessed March 19, 2019].

[11] https://www.poclain-hydraulics.com/ upload/ressources/media/ pdf/A51874K.pdf, [Accessed June 13, 2017]. 